\title{
3D-MODELING OF VEGETATION FROM LIDAR POINT CLOUDS AND ASSESSMENT OF ITS IMPACT ON FAÇADE SOLAR IRRADIATION
}

\author{
G. Peronato ${ }^{\mathrm{a}, *}$ E. Rey ${ }^{\mathrm{b}}$, M. Andersen ${ }^{\mathrm{a}}$ \\ ${ }^{a}$ Interdisciplinary Laboratory of Performance-Integrated Design (LIPID) \\ ${ }^{\mathrm{b}}$ Laboratory of Architecture and Sustainable Technologies (LAST) \\ (giuseppe.peronato, emmanuel.rey, marilyne.andersen)@epfl.ch \\ Ecole polytechnique fédérale de Lausanne (EPFL), Lausanne, Switzerland
}

KEY WORDS: LiDAR, solar potential, solar irradiation, vegetation, convex hull, individual tree crown detection (ITCD)

\begin{abstract}
:
The presence of vegetation can significantly affect the solar irradiation received on building surfaces. Due to the complex shape and seasonal variability of vegetation geometry, this topic has gained much attention from researchers. However, existing methods are limited to rooftops as they are based on 2.5D geometry and use simplified radiation algorithms based on view-sheds. This work contributes to overcoming some of these limitations, providing support for 3D geometry to include facades. Thanks to the use of ray-tracing-based simulations and detailed characterization of the $3 \mathrm{D}$ surfaces, we can also account for inter-reflections, which might have a significant impact on façade irradiation.

In order to construct confidence intervals on our results, we modeled vegetation from LiDAR point clouds as 3D convex hulls, which provide the biggest volume and hence the most conservative obstruction scenario. The limits of the confidence intervals were characterized with some extreme scenarios (e.g. opaque trees and absence of trees).

Results show that uncertainty can vary significantly depending on the characteristics of the urban area and the granularity of the analysis (sensor, building and group of buildings). We argue that this method can give us a better understanding of the uncertainties due to vegetation in the assessment of solar irradiation in urban environments, and therefore, the potential for the installation of solar energy systems.
\end{abstract}

\section{INTRODUCTION}

The growing use of buildings as decentralized renewable energy sources reinforces the need for methods providing a quantification of the energy potential from solar systems in existing urban areas. The IEA (2002, p. 10) estimated that façades can provide about $15-20 \%$ of the electricity production from buildingintegrated photovoltaics. However, façades in urban areas are highly affected by shading and reflection from the surrounding elements, typically other buildings and vegetation.

Previous studies have shown how vegetation influences solar irradiation on rooftops (Tooke et al., 2011; Levinson et al., 2009; Fogl and Moudrý, 2016), also taking into account the semitransparent nature of tree canopies (Tooke et al., 2012). However, these studies use 2.5D models, which limit the application to rooftops and not to façades, unless these are assumed contiguous to the roofs and a hyper-point approach is used (Catita et al., 2014). Moreover, these studies did not include inter-reflections, which might significantly contribute to the total façade irradiation in urban environments.

LiDAR data have been extensively used in recent years for automatic Individual Tree Crown Detection (ITCD), but only a small fraction of studies focused on shape reconstruction (Zhen et al., 2016). Convex hull algorithms have been applied for this scope (Gupta et al., 2010), while voxel-based techniques have been implemented for segmenting tree crowns (Wang et al., 2008).

This exploratory study describes a method to create a 3D representation of trees from LiDAR point clouds using a convex hull algorithm, as part of the simulation of the solar potential of an urban 3D model. We show its application to calculate hourly solar

\footnotetext{
${ }^{*}$ Corresponding author
}

irradiances on building surfaces. By simulating three scenarios of vegetation surface characteristics (reflectivity and transmissivity), we represent three distinct conditions of the seasonal variability of deciduous vegetation.

An exact representation of each type of vegetation cannot be targeted at present, mostly because of the lack of appropriate datasets representing the variability of tree canopies over time. Therefore, this method focuses instead on the uncertainty that has to be considered while analyzing solar irradiation if the characteristics of single trees (deciduous or evergreen, transmissivity and reflectivity of foliage, etc.) are not known.

\section{METHODOLOGY}

The processed datasets consist of (a) vegetation 3D-points from a LiDAR dataset (7-14 p/ $\left.\mathrm{m}^{2}\right)$, (b) a Digital Terrain Model $(0.5 \mathrm{~m}$ resolution) and (c) a 3D vector model of buildings (LOD1). The work-flow is composed of two main phases, as detailed below. Tiled data (350x350 m grid with a $50 \mathrm{~m}$ overlap on each side) produced in a GIS platform is imported and processed within the Rhinoceros ${ }^{1} \mathrm{CAD}$ environment through the Grasshopper ${ }^{2}$ visual programming platform, coupled with different plug-ins and external programs.

Three tiles extracted from the city of Neuchâtel $\left(46^{\circ} 59^{\prime} \mathrm{N}\right.$ $6^{\circ} 56^{\prime} \mathrm{E}, 430 \mathrm{~m}$ asl), whose main morphological characteristics are listed in Table 1, comprise the case study for this paper.

\subsection{Tree segmentation and shape reconstruction}

The tree segmentation and shape reconstruction phases are based respectively on voxel and convex-hull algorithms, which have

\footnotetext{
${ }^{1}$ http://www.rhino3d.com

${ }^{2}$ http://www.grasshopper3d.com
} 

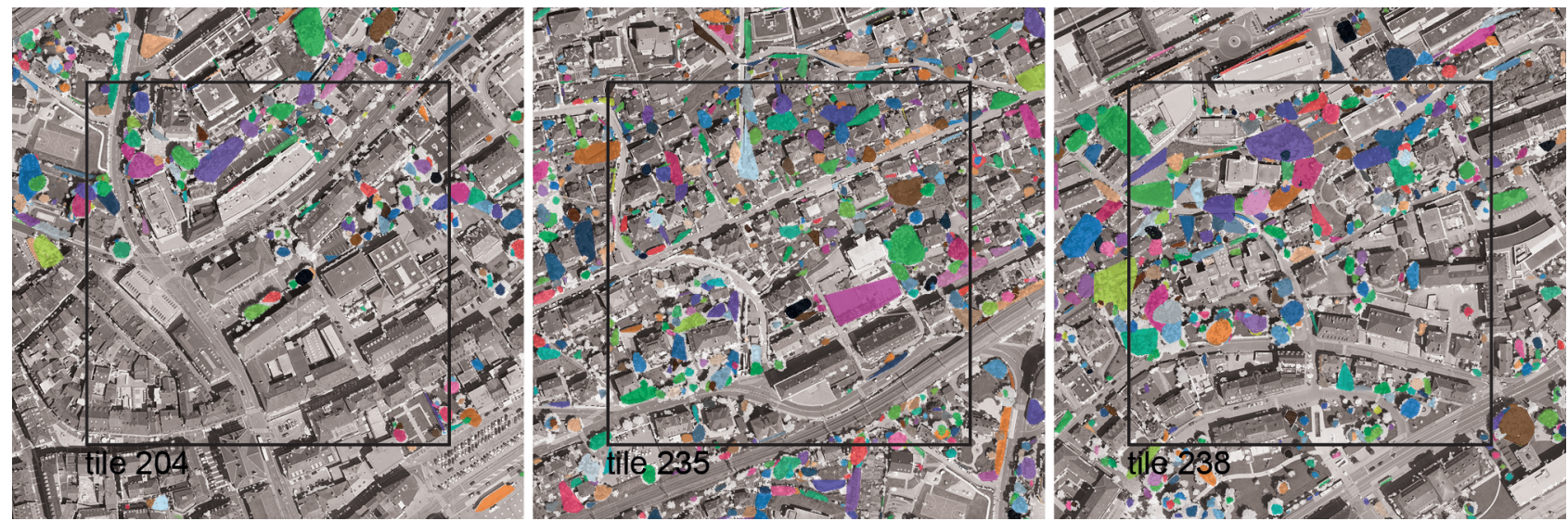

Figure 1. The three selected urban areas (interior square $=250 \mathrm{~m}$ ) with in colors the segmented tree crowns, in white the filtered points.

Table 1. Green and built volume, calculated in a raster $2.5 \mathrm{D}$ model. The tiles IDs refer to Figure 1.

\begin{tabular}{lrrr}
\hline Tile & \multicolumn{1}{c}{204} & \multicolumn{1}{c}{235} & \multicolumn{1}{c}{238} \\
\hline Vegetation $\left[\mathrm{m}^{3}\right]$ & 55222 & 91814 & 108560 \\
Buildings $\left[\mathrm{m}^{3}\right]$ & 455099 & 161460 & 192226 \\
Ratio [-] & 0.12 & 0.57 & 0.56 \\
\hline
\end{tabular}

been already used in the literature for ITCD (e.g. Wang et al., 2008; Gupta et al., 2010). A convex hull describes the biggest volume including the vegetation points, so it is particularly suitable to produce a scenario representing the maximum tree obstruction. The choice of these algorithms is also motivated by the fact that they are included in existing Grasshopper plugins or can be easily integrated in the workflow through coupling with external programs.

The LiDAR vegetation points are first processed using the Volvox plugin ${ }^{3}$ for Grasshopper (see Fig. 1). The points are divided into several point clouds using a voxel topology of $50 \mathrm{~cm}$, which is within the optimal size range suggested by Wang et al. (2008) for LiDAR data of density between 5 and $12 \mathrm{p} / \mathrm{m}^{2}$. To filter out the noise due to vegetation points, only clouds with more than 50 points are kept. Finally, the point clouds are processed in the QHull engine (Barber et al., 1996) to produce the convex hulls representing the trees.

\subsection{Simulation}

An annual simulation of solar radiation is conducted in Radiance/Daysim (Reinhart and Walkenhorst, 2001), through the Ladybug/Honeybee interface (Roudsari and Pak, 2013). The simulation inputs are the sensor grid, the geometry (buildings, vegetation and terrain meshes) and the material definitions. The terrain mesh is created through a Delaunay triangulation using the DTM data. During the ray-tracing, one reflection of each ray is taken into account by setting the number of permissible ambient bounces (-ab parameter) to 2 . An evenly-spaced sensor grid of $1 \mathrm{x} 1 \mathrm{~m}$ is created on all building surfaces. Building surfaces and terrain are defined as Lambertian diffusers with 0.30 and 0.10 reflectivity respectively, as suggested by IESNA (2012), while for vegetation the following scenarios are used:

0. Trees are not included, to model an extreme situation with trees without leaves and trunk.

\footnotetext{
${ }^{3} \mathrm{http} / / / \mathrm{www} . g r a s s h o p p e r 3 d . c o m / g r o u p / v o l v o x$
}

1. Trees are modeled with a translucent material, with 0.553 reflectivity and 0.19 transmissivity. This settings are suggested by Radiance main developer Greg Ward, as cited by Jakubiec and Balakrishnan (2015).

2. Trees are modeled with an opaque material with 0.20 reflectivity, as suggested by by IESNA (2012).

It should be noted that the time for simulating scenario 2 is significantly longer (up to 10 times) than the one for the other two. Also, because ambient bounces are limited to 2, only direct solar radiation passes through the vegetation canopy.

\section{RESULTS}

As can be seen in Table 2, the maximum annual variation (scenario 1 - scenario 2) for all tiles is of about $860 \mathrm{kWh} / \mathrm{m}^{2}$. This corresponds to the maximum irradiation that can be achieved on façades, and that can be completely lost due to the obstruction of vegetation (scenario 1). Minimum values are also similar and negative for all façades. This is probably due to the reflection of trees on North-exposed façades. The upper and lower quartile boundaries show that the variation in this range is much smaller. We also notice that while the lower and median quartiles have similar values, the upper quartile significantly changes depending on the characteristics of the tile.

If we look at the results of single buildings, for example those of tile 204 in Figure 3b, we can see that the variation between buildings is very high, and in some cases can be much higher than the variation of the whole tile. In our findings, exposed buildings are more sensitive to the vegetation effects. This can be seen in the results of building 23, which has large South-exposed façades.

Table 2. Asolute variation of annual solar irradiation per sensor point. Values are expressed in $\mathrm{kWh} / \mathrm{m}^{2}$. The tile IDs refer to Figure 1.

\begin{tabular}{lcrcrc}
\hline Pecentile & 0 & \multicolumn{1}{c}{25} & \multicolumn{1}{c}{50} & \multicolumn{1}{c}{75} & 100 \\
\hline tile 204 & -40.39 & -0.13 & 0.63 & 6.66 & 857.60 \\
tile 235 & -43.59 & 0.01 & 5.28 & 29.57 & 848.38 \\
tile 238 & -34.74 & 0.73 & 8.63 & 53.52 & 862.71 \\
\hline
\end{tabular}

If we look at the monthly values (Figure $4 a$ ), we see a similar seasonal trend for all scenarios. In particular, scenario 1 is always between the curves of the other two scenarios. In relative terms, the difference between scenario 0 and 2 (Figure $4 \mathrm{~b}$ ) shows a different trend, with peaks in winter and in summer. 


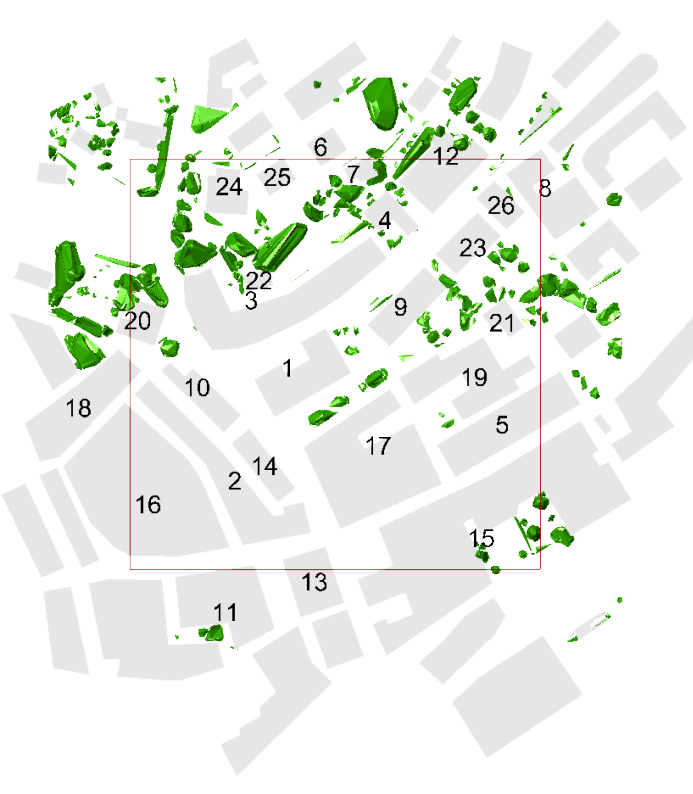

Figure 2. Plan of tile 204. The simulated sensor points are contained in the square.

\section{DISCUSSION}

The results of the analyzed tiles fall within the ranges of relative variation of solar irradiation due to vegetation (3-20\%) obtained by previous studies focusing on rooftops (Levinson et al., 2009; Fogl and Moudrý, 2016). In absolute values, the results are not directly comparable, because of the different climatic conditions as well as by the fact that our analysis was targeted to façades.

By using a higher level of spatial granularity than previous studies, we also showed that the value for the entire tile is not representative of the conditions of the different buildings and building surfaces belonging to it, at least in our case study, which has an irregular distribution of buildings and vegetation. In this sense, the method could have an useful application also for building owners, who would be able to evaluate the risk of irradiation loss due to vegetation in the different parts of the building envelope. The high spatial variability also suggests that the results cannot be extrapolated to different urban areas (e.g. many suburban developments where there is a regular arrangement of buildings and trees) nor climates. However, by increasing the number of simulated tiles, we would be able to check whether some geometric indicators such as the vegetation volume can predict the uncertainty on the calculated irradiation. This would help reduce the number of simulations of tree scenarios to only the tiles and/or the building in which vegetation has a significant impact.

Regarding the semi-transparent nature of trees, we have seen that the results of scenario 1 are always within the values of senario 0 and scenario 2 . Therefore, we can argue that these latter scenarios provide a better description of the uncertainty that has to be considered while assessing the solar potential of an urban area. Moreover, the lower simulation time makes these scenarios more suitable for large-scale assessments.

\section{LIMITATIONS AND OUTLOOK}

In this work, we limited the analysis to the façades, as the LOD 1 model was considered inaccurate for assessing the results of rooftops (Peronato et al., 2016). We plan to extend this study to the whole building envelope using a LOD 2.3 model, which,

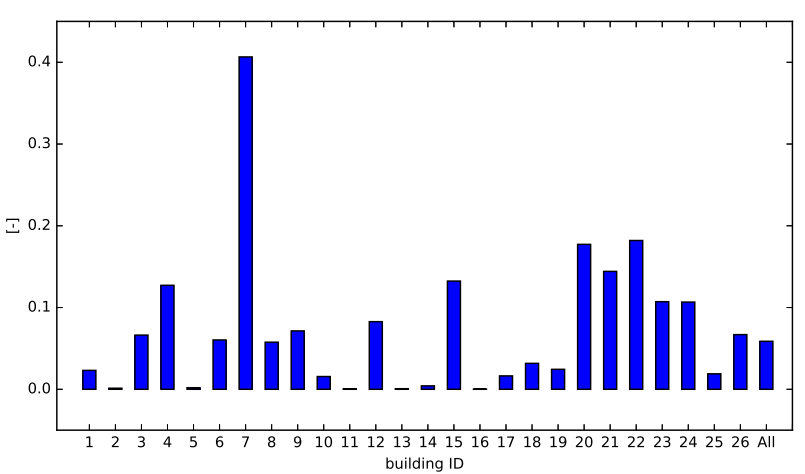

(a) Relative values per building.

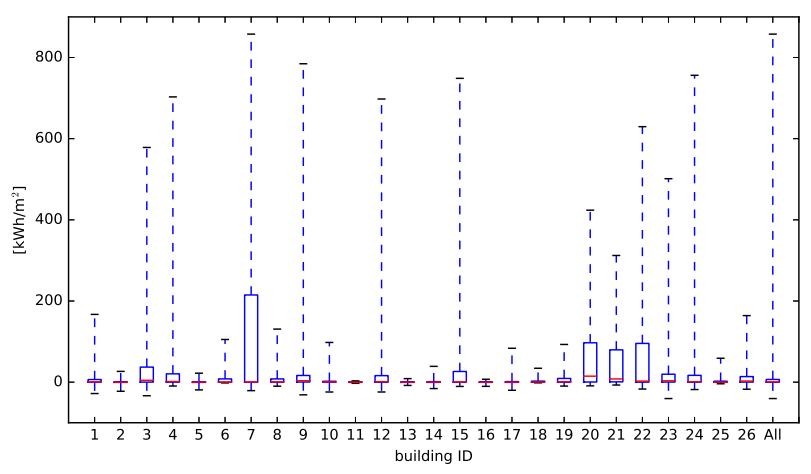

(b) Absolute values per sensor point.

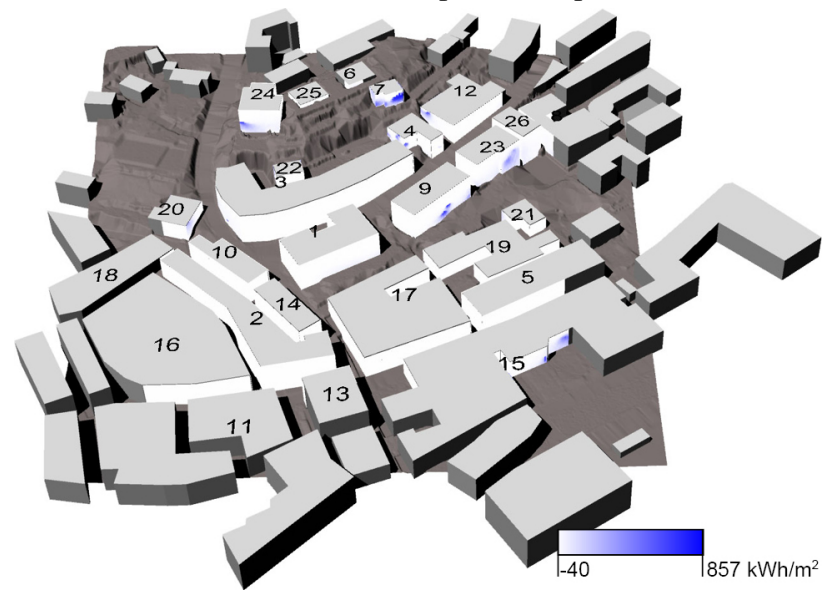

(c) Absolute values per sensor point in false colors. Vegetation is considered but not shown.

Figure 3. Difference of annual solar irradiation between scenario 0 and scenario 2 (tile 204).

according to the categorization proposed by Biljecki et al. (2016), includes the actual roof shape and rooftop superstructures.

The 3D-representation of vegetation is not always very accurate, mostly due to the errors in the segmentation phase when considering contiguous trees (see Figure 1). We plan to test other algorithms/parameters and other datasets to improve the reliability and robustness of the method.

\section{CONCLUSIONS}

The method proposed by this work can help decision-makers compare different solar energy planning scenarios accounting for 


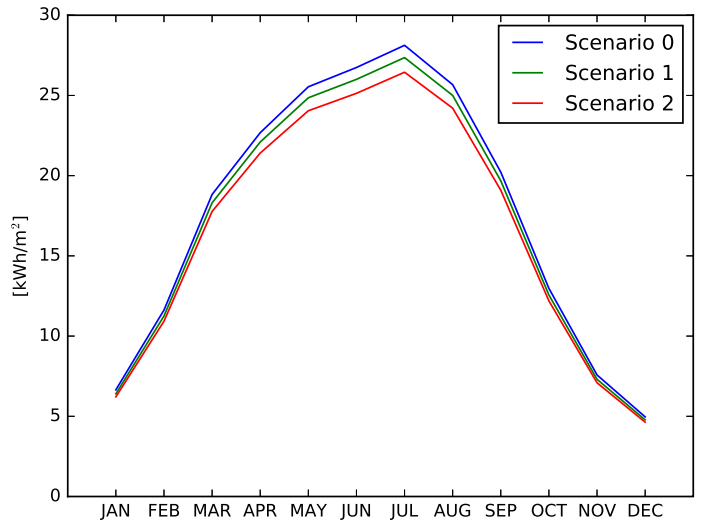

(a) Absolute values for the three scenarios.

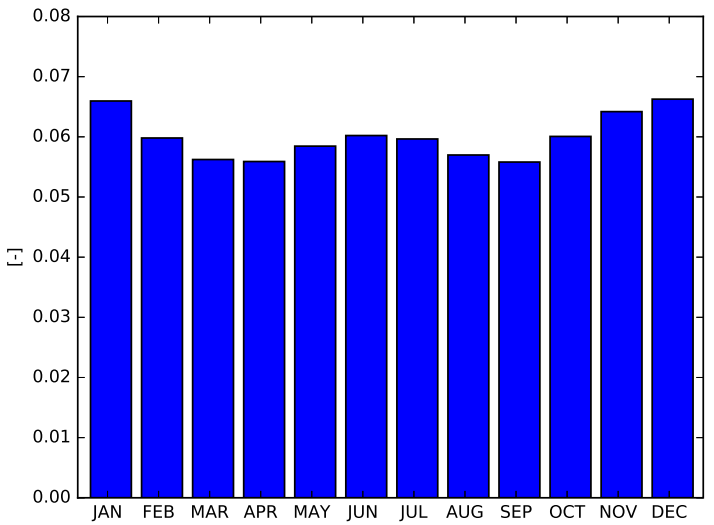

(b) Relative difference between scenario 0 and scenario 2 .

Figure 4. Monthly solar irradiation (tile 204).

the uncertainty due to vegetation. Although the absolute reduction of irradiation on façades found in the case-study application is rather low (median annual reduction of $1-9 \mathrm{kWh} / \mathrm{m}^{2}$ ), in relative terms the results are comparable to those obtained by precedent studies on rooftops (Levinson et al., 2009; Fogl and Moudrý, 2016). Moreover, by augmenting the granularity of the analysis at the building scale, we have seen that the reduction can be very significant (up to $40 \%$ for a single building), depending on the solar exposure and the presence of obstructing vegetation.

\section{ACKNOWLEDGEMENTS}

This work has been conducted in the framework of the ACTIVE INTERFACES research project (Rey et al., 2015), which is part of the National Research Programme "Energy Turnaround" (NRP 70) of the Swiss National Science Foundation (SNSF). Further information on the National Research Programme can be found at http://www.nrp70.ch.

The geodata used in this work have been kindly provided by the Service Information du Territoire Neuchâtelois (c)2015 Service de la Géomatique et du Registre Foncier) and by the Swiss Federal Office of Topography (C)2010 Swisstopo).

\section{References}

Barber, C. B., Dobkin, D. P. and Huhdanpaa, H., 1996. The Quickhull Algorithm for Convex Hulls. ACM Trans. Math. Softw. 22(4), pp. 469-483.

Biljecki, F., Ledoux, H. and Stoter, J., 2016. An improved LOD specification for $3 \mathrm{~d}$ building models. Computers, Environment and Urban Systems 59, pp. 25-37.

Catita, C., Redweik, P., Pereira, J. and Brito, M. C., 2014. Extending solar potential analysis in buildings to vertical facades. Computers \& Geosciences 66, pp. 1-12.

Fogl, M. and Moudrý, V., 2016. Influence of vegetation canopies on solar potential in urban environments. Applied Geography 66, pp. 73-80.

Gupta, S., Weinacker, H. and Koch, B., 2010. Comparative Analysis of Clustering-Based Approaches for 3-D Single Tree Detection Using Airborne Fullwave Lidar Data. Remote Sensing 2(4), pp. 968-989.

IEA, 2002. Potential for Building Integrated Photovoltaics - Full Technical Report. Technical Report PVPS T7- 4.

IESNA, 2012. IES LM-83-12 IES Spatial Daylight Autonomy (sDA) and Annual Sunlight Exposure (ASE). Technical Report IES LM-83-12, New York, NY, USA.

Jakubiec, J. A. and Balakrishnan, P., 2015. Quantifying Materials in Lighting Simulations. 14th International Radiance Workshop, Philadephia, USA.

Levinson, R., Akbari, H., Pomerantz, M. and Gupta, S., 2009. Solar access of residential rooftops in four California cities. Solar Energy 83(12), pp. 2120-2135.

Peronato, G., Bonjour, S., Stoeckli, J., Rey, E. and Andersen, M., 2016. Sensitivity of calculated solar irradiation to the level of detail: insights from the simulation of four sample buildings in urban areas. 32nd International PLEA Conference, Los Angeles, USA.

Reinhart, C. F. and Walkenhorst, O., 2001. Validation of dynamic RADIANCE-based daylight simulations for a test office with external blinds. Energy and Buildings 33(7), pp. 683-697.

Rey, E., Lufkin, S., Ballif, C., Wuestenhagen, R., Wittkopf, S. and Bacher, J.-P., 2015. Building integrated photovoltaics | ACTIVE INTERFACES.

Roudsari, M. S. and Pak, M., 2013. Ladybug: a parametric environmental plugin for grasshopper to help designers create an environmentally-conscious design. In: Proceedings of the 13th International IBPSA Conference.

Tooke, T. R., Coops, N. C., Christen, A., Gurtuna, O. and Prévot, A., 2012. Integrated irradiance modelling in the urban environment based on remotely sensed data. Solar Energy 86(10), pp. 2923-2934.

Tooke, T. R., Coops, N. C., Voogt, J. A. and Meitner, M. J., 2011. Tree structure influences on rooftop-received solar radiation. Landscape and Urban Planning 102(2), pp. 73-81.

Wang, Y., Weinacker, H. and Koch, B., 2008. A Lidar Point Cloud Based Procedure for Vertical Canopy Structure Analysis And 3d Single Tree Modelling in Forest. Sensors 8(6), pp. 3938-3951.

Zhen, Z., Quackenbush, L. J. and Zhang, L., 2016. Trends in Automatic Individual Tree Crown Detection and Delineation-Evolution of LiDAR Data. Remote Sensing 8(4), pp. 333. 